\title{
Dust Formation in Stellar Winds of Very Massive Population III Stars
}

\section{Takaya Nozawa*}

National Astronomical Observatory of Japan, Japan, E-mail: takaya . nozawa@nao . ac . jp

\section{Takashi Kozasa}

Department of Cosmosciences, Graduate School of Science, Hokkaido University, Japan

\section{Sung-Chul Yoon}

Department of Physics and Astronomy, Seoul National University, Korea

\section{Keiichi Maeda}

Department of Astronomy, Kyoto University, Japan

\section{Norbert Langer}

Argelander-Institut für Astronomie der Universität Bonn, Germany

\section{Ken'ichi Nomoto}

Kavli Institute for the Physics and Mathematics of the Universe (WPI), The University of Tokyo, Japan

\begin{abstract}
The origin of dust grains in the early universe is one of the most challenging subjects in astrophysics. Recent study of the evolution of massive Population III stars has suggested that the metal-enriched mass-loss winds from very massive Population III red-supergiants with the initial masses higher than $M_{\text {ZAMS }} \simeq 250 M_{\odot}$ could be potential formation sites of dust grains. In this proceedings, in order to explore the possibility of dust formation in such stellar winds, we present the analytical formulae that describe the formation condition of dust grains in steady stellar winds. We find that, in a stellar wind of a Population III red-supergiant with $M_{\text {ZAMS }}=500 M_{\odot}$, the formation of carbon grains is possible if the mass-loss rate and wind velocity satisfy the condition $\left(\dot{M} / 10^{-5} M_{\odot} \mathrm{yr}^{-1}\right)\left(v_{\mathrm{w}} / 10 \mathrm{~km} \mathrm{~s}^{-1}\right)^{-2} \gtrsim 0.06$. We note that a series of formulae given in this study would be useful in evaluating the feasibility of dust formation in steady mass-loss winds of various types of stellar populations.
\end{abstract}

The Life Cycle of Dust in the Universe: Observations, Theory, and Laboratory Experiments 18-22 November, 2013

Taipei, Taiwan

\footnotetext{
*Speaker.
} 


\section{Introduction}

The first cosmic dust is considered to be produced in the metal-rich ejecta of supernovae (SNe) that occur from the first stellar generation, so-called Population III (Pop III) stars. Numerical simulations of the formation of metal-free stars have shown that the mass of Pop III stars would be much higher than those of the local Pop I/II stars [1, 2], supporting that SNe arising from massive stars would play dominant roles in dust enrichment history of the early universe. However, a typical mass of Pop III stars is a matter of controversy; the predicted mass-scale ranges from a few tens $M_{\odot}$ $[3,4]$ up to more than a few hundreds $M_{\odot}[5,6]$. If Pop III stars have formed with the initial masses above $M_{\text {ZAMS }} \simeq 250 M_{\odot}$, they are likely to finally collapse into black holes and fail to disperse a large amount of heavy elements at the end of their lives [7, 8].

On the other hand, such very massive Pop III stars could chemically enrich the surrounding medium during their evolutionary stages. Yoon et al. [8] extensively investigated the evolution of massive Pop III stars for the mass range of 10-1000 $M_{\odot}$, and found that non-rotating stars with $M_{\text {ZAMS }} \gtrsim 250 M_{\odot}$ experience convective dredge-up of a significant amount of carbon and oxygen from the helium-burning core into the hydrogen envelope at the red-supergiant (RSG) phase. Thus, if the mass loss is driven efficiently during the RSG phase, the CNO-enriched stellar winds can pollute the ambient medium and can also offer formation sites of the first dust grains in the universe.

In this proceedings, with the aim of assessing the possibility of dust formation in RSG winds of very massive Pop III stars, we present the analytical formulae describing the formation condition of carbon grains in steady stellar winds. The formula is based on the scaling relation of dust formation given in Nozawa \& Kozasa [9] and would be very useful in evaluating the feasibility of dust formation in mass-loss winds of any kinds of stellar populations.

\section{The Model}

Recently, Nozawa \& Kozasa [9] formulated the non-steady-state formation of small clusters and the growth of grains accompanied by chemical reactions. By examining the results of dust formation calculations for various gas densities and cooling rates, they found that the condensation efficiency and average radius of newly formed grains are universally described by a non-dimensional quantity $\Lambda_{\text {on }}$ defined as the ratio of the supersaturation timescale $\tau_{\text {sat }}$ to the collision timescale $\tau_{\text {coll }}$ at the onset time $t_{\mathrm{on}}$ of dust formation. They also showed that the formation of $\mathrm{C}$ and $\mathrm{MgSiO}_{3}$ grains is realized when $\Lambda_{\text {on }}>1$. Hence, the formation of dust in mass-loss winds can be evaluated by deriving $\Lambda_{\text {on }}$ for given wind properties such as the mass-loss rate and wind velocity.

In general, the mass-loss rate and wind velocity of massive stars are not well known; the mass loss may be caused pulsationally and dynamically as a result of multiple effects of centrifugal force, magnetic fields, turbulence, and outburst in stellar surface. Thus, it might be hard to predict accurately the total amount and size distribution of dust formed in the course of the stellar evolution. In this study, instead of modeling the detailed mass-loss history, we aim at presenting the ranges of the mass-loss rate and wind velocity with which the formation of dust is feasible in stellar winds. In the following, we describe the model of density, temperature, and chemical composition of the outflowing gas, and demonstrate how $\Lambda_{\text {on }}$ is expressed by the mass-loss rate and wind velocity. 


\subsection{Hydrodynamic Model of the Circumstellar Gas}

We consider a simple model where the circumstellar gas is a spherically symmetric outflow with a constant wind velocity. In this case, the density profile of the outflowing gas is given by

$$
\rho(r)=\frac{\dot{M}}{4 \pi r^{2} v_{\mathrm{w}}}=\frac{\dot{M}}{4 \pi R_{\text {star }}^{2} v_{\mathrm{w}}}\left(\frac{r}{R_{\text {star }}}\right)^{-2},
$$

where $\dot{M}$ is the mass-loss rate, $v_{\mathrm{w}}$ is the wind velocity, $R_{\text {star }}$ is the stellar radius, and $r$ is the distance from the center of the star. The temperature distribution of the gas is assumed to be

$$
T(r)=T_{\text {star }}\left(\frac{r}{R_{\text {star }}}\right)^{-\alpha}
$$

where $T_{\text {star }}$ is the effective temperature of the star, and $\alpha \simeq 1$ for adiabatic expansion, and $\alpha=0.5$ for the case of radiative equilibrium.

\subsection{Chemical Composition of the Circumstellar Gas}

As mentioned in the Introduction, very massive Pop III stars with $M_{\text {ZAMS }} \gtrsim 250 M_{\odot}$ could undergo extensive dredge-up during helium-core burning, which conveys a considerable amount of carbon and oxygen to the hydrogen envelope [8]. For the model of a $500 M_{\odot}$ RSG without rotation, the average number fractions of carbon and oxygen in the outer envelope are $A_{\mathrm{C}}=3.11 \times 10^{-3}$ and $A_{\mathrm{O}}=1.75 \times 10^{-3}$, respectively [8]. Thus, the gas outflowing from very massive RSGs is expected to be carbon-rich with a high $\mathrm{C} / \mathrm{O}$ ratio $\left(A_{\mathrm{C}} / A_{\mathrm{O}}=1.78\right.$ for the $500 M_{\odot} \mathrm{RSG}$ model).

In a carbon-rich cooling gas, all oxygen atoms react with carbon atoms to form $\mathrm{CO}$ molecules, and carbon atoms and/or carbon-bearing molecules left after the $\mathrm{CO}$ formation are available for the formation of carbon clusters and grains. Figure 1 shows the result of the chemical equilibrium calculation along the gas flow [10], as an example, for the $500 M_{\odot}$ Pop III RSG (see below for the properties of this star). It can be seen that $\mathrm{CO}$ molecule is the stable gas species in the temperature range considered here, and most of the carbon unbound in $\mathrm{CO}$ molecules exists as atomic carbon at $T>1750 \mathrm{~K}$ and as $\mathrm{C}_{2} \mathrm{H}$ at $1400 \mathrm{~K}<T<1700 \mathrm{~K}$. This indicates that the formation of carbon grains is likely to proceed through successive attachment of carbon atoms (or $\mathrm{C}_{2} \mathrm{H}$ molecules) at high temperatures.

\subsection{Derivation of the non-dimensional quantity $\Lambda_{\mathrm{on}}$}

For the wind model given above, we here provide a non-dimensional quantity $\Lambda_{\text {on }}=\tau_{\text {sat }} / \tau_{\text {coll }}$, considering that carbon grains condense via the successive attachment of carbon atoms.

The timescale of collisions of carbon atoms is given by

$$
\tau_{\text {coll }}^{-1}=\pi a_{0}^{2}\left(\frac{8 k T}{\pi m_{\mathrm{C}}}\right)^{\frac{1}{2}} \tilde{n}_{\mathrm{C}}
$$

where $a_{0}$ is the radius of the condensate per atom, $k$ is the Boltzmann constant, $m_{\mathrm{C}}$ is the mass of a carbon atom, and $\tilde{n}_{\mathrm{C}}$ is the nominal number density of free carbon atoms unbound in $\mathrm{CO}$ molecules (see Section 2.3 in Nozawa \& Kozasa [9] for the definition of the nominal number density). By 


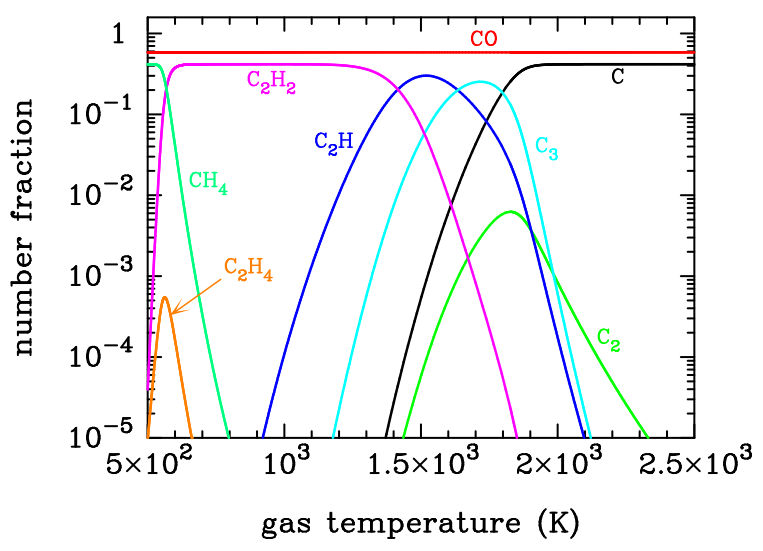

Figure 1: Number fractions of carbon-bearing gas species as a function of gas temperature obtained from the chemical equilibrium calculation for the circumstellar envelope model of the $500 M_{\odot}$ Pop III RSG.

introducing a parameter $f=A_{\mathrm{C}} / A_{\mathrm{O}}$, which is the number ratio of carbon atoms to oxygen atoms, the nominal number density of carbon atoms is written as

$$
\tilde{n}_{\mathrm{C}}=\frac{A_{\mathrm{C}}}{\mu m_{\mathrm{H}}}\left(1-\frac{1}{f}\right)\left(\frac{\dot{M}}{4 \pi R_{\mathrm{star}}^{2} v_{\mathrm{w}}}\right)\left(\frac{T}{T_{\mathrm{star}}}\right)^{\frac{2}{\alpha}},
$$

where $\mu$ is the mean molecular weight, and $m_{\mathrm{H}}$ is the mass of hydrogen. The supersaturation ratio $S$ is described by

$$
\ln S=-\frac{\Delta \stackrel{\circ}{k T}}{k T} \ln \left(\frac{\tilde{n}_{\mathrm{C}} k T}{p_{\mathrm{v}}}\right)=\frac{\gamma}{T}-\delta+\ln \left(\frac{\tilde{n}_{\mathrm{C}} k T}{p_{\mathrm{v}}}\right),
$$

where $p_{\mathrm{v}}$ is the vapor pressure of the condensate, and the Gibbs free energy for the formation of bulk condensate from the vapor is approximated by $\Delta \stackrel{g}{ } / k T=-\gamma / T+\delta$ with the numerical values $\gamma$ and $\delta$ derived by least-squares fittings of the thermodynamics data [11] in the temperature range of $T=200-2500 \mathrm{~K}$. Then, the timescale on which the supersaturation ratio increases is given as

$$
\tau_{\mathrm{sat}}^{-1}=\frac{d \ln S}{d t}=\frac{\alpha v_{\mathrm{w}}}{R_{\mathrm{star}}}\left(\frac{T}{T_{\mathrm{star}}}\right)^{\frac{1}{\alpha}}\left(\frac{\gamma}{T}-\frac{2}{\alpha}-1\right) .
$$

From Equations (2.3) and (2.6), the non-dimensional quantity $\Lambda_{\text {on }}$ is written as

$$
\Lambda_{\mathrm{on}}=\left.\frac{\tau_{\mathrm{sat}}}{\tau_{\mathrm{coll}}}\right|_{t=t_{\mathrm{on}}}=\frac{A_{\mathrm{C}} \pi a_{0}^{2}}{\alpha \mu m_{\mathrm{H}}}\left(1-\frac{1}{f}\right)\left(\frac{8 k T_{\mathrm{on}}}{\pi m_{\mathrm{C}}}\right)^{\frac{1}{2}}\left(\frac{\dot{M}}{4 \pi R_{\mathrm{star}} v_{\mathrm{w}}^{2}}\right)\left(\frac{T_{\mathrm{on}}}{T_{\mathrm{star}}}\right)^{\frac{1}{\alpha}}\left(\frac{\gamma}{T_{\mathrm{on}}}-\frac{2}{\alpha}-1\right)^{-1}
$$

where $T_{\mathrm{on}}$ is the gas temperature at $t=t_{\mathrm{on}}$ (that is, condensation temperature of dust). In general, $\alpha=0.5$ is used for the temperature profile of the outflowing gas [e.g., 12]. In this case,

$$
\Lambda_{\mathrm{on}} \simeq 150\left(1-\frac{1}{f}\right)\left(\frac{A_{\mathrm{C}}}{10^{-3}}\right)\left(\frac{\dot{M}}{10^{-5} M_{\odot} \mathrm{yr}^{-1}}\right)\left(\frac{v_{\mathrm{w}}}{10 \mathrm{~km} \mathrm{~s}^{-1}}\right)^{-2}\left(\frac{L_{\mathrm{star}}}{10^{5} L_{\odot}}\right)^{-\frac{1}{2}},
$$

where $L_{\text {star }}$ is the luminosity of the star $\left(=4 \pi R_{\text {star }}^{2} \sigma T_{\text {star }}^{4}\right), a_{0}=1.28 \AA$, and $\gamma=8.64 \times 10^{4} \mathrm{~K}$ [13], and we adopt $\mu=1.3$ and $T_{\text {on }}=1800 \mathrm{~K}$. Equation (2.8) demonstrates that, for $\alpha=0.5$, the formation process of dust (represented by $\Lambda_{\text {on }}$ ) in stellar winds is sensitive to the mass-loss rate $\dot{M}$ and wind velocity $v_{\mathrm{w}}$ but does not depend explicitly on $T_{\mathrm{star}}$. 


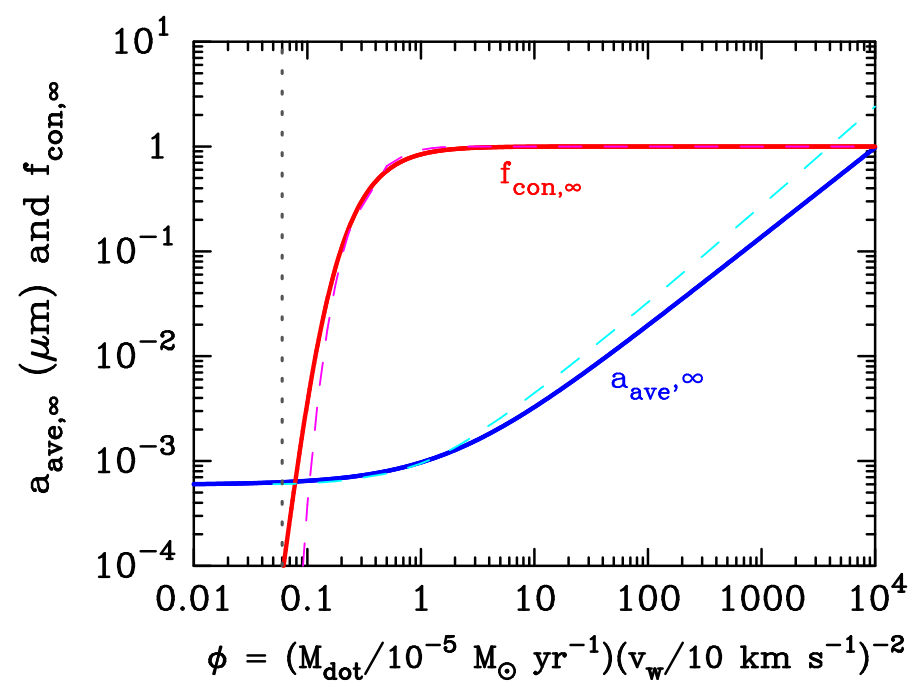

Figure 2: Final average radius $a_{\mathrm{ave}, \infty}$ and condensation efficiency $f_{\text {con, } \infty}$ of carbon grains that can form in a RSG wind of a Pop III star with $M_{\text {ZAMS }}=500 M_{\odot}$ as a function of $\phi$ defined in Equation (3.1). The solid lines are the results obtained analytically from Equations (2.8), (3.2), and (3.3), while the dashed lines are the results of detailed numerical simulations of dust formation. The vertical dotted line marks $\phi=0.06$, above which the formation of dust is found to be possible [see Equation (3.1)].

\section{Formation Condition of Dust in a RSG Wind of a Pop III $500 \mathrm{M}_{\odot}$ Star}

As shown in Nozawa \& Kozasa [9], the formation of carbon grains is possible when $\Lambda_{\text {on }}>1$. Thus, from Equation (2.8), the formation condition for carbon grains in a steady RSG wind of a Pop III $500 M_{\odot} \operatorname{star}\left(f=1.78, A_{\mathrm{C}}=3.11 \times 10^{-3}, L_{\mathrm{star}}=10^{7.2} L_{\odot}\right)$ is given as follows:

$$
\phi=\left(\frac{\dot{M}}{10^{-5} M_{\odot} \mathrm{yr}^{-1}}\right)\left(\frac{v_{\mathrm{w}}}{10 \mathrm{~km} \mathrm{~s}^{-1}}\right)^{-2} \gtrsim 0.06 .
$$

This equation indicates that, for a constant wind velocity of $20 \mathrm{~km} \mathrm{~s}^{-1}$, carbon grains can condense if $\dot{M} \gtrsim 2.5 \times 10^{-6} M_{\odot} \mathrm{yr}^{-1}$.

Nozawa \& Kozasa [9] also presented the approximation formula describing the average grain radius $a_{\text {ave, } \infty}$ and condensation efficiency $f_{\text {con, } \infty}$ as a function of $\Lambda_{\text {on }}$; the approximation formulae for carbon grains are given by

$$
\log \left(\frac{a_{\mathrm{ave}, \infty}}{a_{*}}-1\right)=-1.21+0.854 \log \Lambda_{\mathrm{on}}
$$

and

$$
\log f_{\text {con, } \infty}=4.15\left[\tanh \left(1.91 \log \Lambda_{\text {on }}+0.088\right)-1\right]
$$

where $a_{*}=5.946 \AA$. The solid lines in Figure 2 shows $a_{\text {ave }, \infty}$ and $f_{\text {con, }, \infty}$ obtained from the above analytic formulae as a function of $\phi$ for the $500 M_{\odot}$ Pop III RSG model. The condensation efficiency rapidly increases from $\phi \simeq 0.06$, reaching unity at $\phi \simeq 1$. The dashed lines in Figure 2 
plot the results of the numerical simulations of non-steady-state dust formation, which are in good agreement with the analytical ones. In fact, there are small differences in $a_{\mathrm{ave}, \infty}$ at $\phi \gtrsim 10$ and in $f_{\text {con, } \infty}$ at $\phi \lesssim 0.2$, both of which mainly come from the simple assumption of $T_{\text {on }}=1800 \mathrm{~K}$ in the present analysis; in reality, $T_{\mathrm{on}}$ is higher than $1800 \mathrm{~K}$ for a higher $\Lambda_{\mathrm{on}}$, and vice versa.

In this study, we have put the constraints on the mass-loss rate and wind velocity with which carbon grains can condense in RSG winds of very massive Pop III stars. Here, it should be emphasized that our analysis can be also applicable for dust formation in mass-loss winds of any types of stellar populations, as long as the mass-loss wind is steady. Therefore, a series of formulae given in this work would be powerful tools in assessing the possibility of dust formation in stellar winds.

\section{Acknowledgments}

This research has been supported by World Premier International Research Center Initiative (WPI Initiative), MEXT, Japan, and by the Grant-in-Aid for Scientific Research of the Japan Society for the Promotion of Science (22684004, 23224004).

\section{References}

[1] V. Bromm and R. B. Larson, The first stars, ARA\&A, 2004 (42) 79

[2] S. Hirano, T. Hosokawa, N. Yoshida, H. Umeda, K. Omukai, G. Chiaki, and H. W. Yorke, One hundred first stars: protostellar evolution and the final mass, ApJ, 2014 (781) 60

[3] T. Hosokawa, K. Omukai, N. Yoshida, and H. W. Yorke, Protostellar feedback halts the growth of the first stars in the universe, Science, 2011 (334) 1250

[4] H. Susa, The mass of the first stars, ApJ, 2013 (773) 185

[5] K. Omukai and F. Palla, Formation of the first stars by accretion, ApJ, 2003 (589) 677

[6] T. Ohkubo, K. Nomoto, H. Umeda, N. Yoshida, and S. Tsuruta, Evolution of very massive Population III stars with mass accretion from pre-main sequence to collapse, ApJ, 2009 (706) 1184

[7] A. Heger, C. L. Fryer, S. E. Woosley, N. Langer, and D. H. Hartmann, How massive single stars end their life, ApJ, 2003 (591) 288

[8] S.-C. Yoon, A. Dierks, and N. Langer, Evolution of massive Population III stars with rotation and magnetic fields, A\&A, 2012 (542) A113

[9] T. Nozawa and T. Kozasa, Formulation of non-steady-state dust formation process in astrophysical environments, ApJ, 2013 (776) 24

[10] T. Kozasa, J. Dorschner, T. Henning, and R. Stognienko, Formation of SiC grains and the $11.3 \mu \mathrm{m}$ feature in circumstellar envelopes of carbon stars, A\&A, 1996 (307) 551

[11] M. W. Chase Jr., C. A. Davis, J. R. Downey Jr., D. J. Frurip, R. A. McDonald, and A. N. Syverud, JANAF Thermochemical Tables, 3rd ed., J. Phys. Chem. Ref. Data Suppl., 1985 (14) 1

[12] H.-P. Gail, R. Keller, and E. Sedlmayr, Dust formation in stellar winds. I - A rapid computational method and application to graphite condensation, A\&A, 1984 (133) 320

[13] T. Nozawa, T. Kozasa, H. Umeda, K. Maeda, and K. Nomoto, Dust in the early universe: dust formation in the ejecta of Population III supernovae, ApJ, 2003 (598) 785 